\title{
Gas Sensor MEMS Platform for Harsh Conditions
}

\author{
A.A.Vasiliev ${ }^{1}$, A.S.Lipilin ${ }^{2}$, A.M.Mozalev ${ }^{3}$, A.S.Lagutin ${ }^{1}$, A.V.Pisliakov ${ }^{1}$, N.P.Zaretskiy ${ }^{1}$, N.N.Samotaev ${ }^{1}$, \\ A. V.Sokolov', S.A.Soloviev ${ }^{1}$ \\ ${ }^{1}$ NRC "Kurchatov Institute", IACPh, Kurchatov sq., 1, 123182, Moscow, Russia \\ ${ }^{2}$ Institute of Electrophysics of Ural Branch of RAS, Amundsen str., 106, 620016, Ekaterinburg, Russia \\ Corresponding e-mail address: A-A-Vasiliev@yandex.ru \\ ${ }^{3}$ Belarus State University of radioelectronics and informatics, P. Brovki str., 6, Minsk, 220013, Belarus
}

\begin{abstract}
We present a novel approach to the fabrication of MEMS devices, which can be used for gas sensors operating in harsh environment in wireless and autonomous information systems. MEMS platforms based on $\mathrm{ZrO}_{2} / \mathrm{Y}_{2} \mathrm{O}_{3}$ (YSZ) and alumina membranes are applied in these devices. The methods of fabrication of these ceramic MEMS devices are considered. It is shown that the application of such membranes permits a decrease in MEMS power consumption at $450^{\circ} \mathrm{C}$ down to $\sim 75 \mathrm{~mW}$ at continuous heating and down to $\sim 1 \mathrm{~mW}$ at pulse heating of gas sensor. The application of the platforms is not restricted by gas sensors: they can be used for fast thermometers, bolometric matrices, flowmeteres and other MEMS devices working under harsh environmental conditions.
\end{abstract}

Key words: gas sensors, harsh environment, wireless and autonomous information systems, ceramic MEMS devices

\begin{abstract}
Introduction
Gas sensors are widely used in the systems of monitoring of industrial atmosphere, environment, and technological process. They operate not only under normal conditions, but also in very harsh environment that is at high temperature, high humidity, in aggressive atmosphere. Harsh industrial and ecological catastrophes of last years, for example the accident in Fukushima, showed that there is no devices, which could operate under such conditions. As a result, actual information about the concentration of hydrogen, toxic gases, humidity etc. is not available for immergence service, and the accident develops following the most dangerous and even tragic scenario.
\end{abstract}

During last years, National research center "Kurchatov institute" paid attention to the development of sensors and sensor systems based on MEMS structures, which could be applied under such conditions. It is important not only to develop the sensors, but also to have a possibility to test these devices in explosive atmosphere, in flame and explosion of gases. This possibility exists in the field testing area of the Institute.

In traditional MEMS instruments fabricated using silicon technology, thin dielectric membrane applied as supporting and thermoinsulating element of sensor is made of several layers of silicon oxide and silicon nitride necessary to decrease mechanical strains in the membrane. Thin dielectric membrane of such sensor is fabricated by successive CVD deposition of silicon oxide and silicon nitride layers on silicon wafer followed by anisotropic etching of silicon from the back side of silicon. This etching releases free dielectric membrane.

Many researchers and companies (for example [1-3]), producing gas sensors, fabricate gas sensors on MEMS silicon based platforms. However, in spite of all effort made in this field, multilayer membranes made of silicon oxide and silicon nitride have now several important drawbacks. (1) Multi-layer dielectric membrane of silicon-based MEMS consists of materials with different thermal and mechanical properties. Therefore, there are strong interlayer stresses in the membrane, which can break the membrane even at room temperature and lead to mechanical fatigue of the membrane under temperature modulation. (2) Platinum and ruthenium dioxide are the only materials of heaters, which are stable at high temperature in oxygen-containing atmosphere. However, adhesion of platinum to silicon oxide and silicon nitride is poor, especially at working (up to $450^{\circ} \mathrm{C}$ ) and annealing $\left(700-800^{\circ} \mathrm{C}\right.$ ) temperature of sensing layer. The application of usual metallic adhesive layers does not improve very much this situation. Some possible technical solutions of this problem are not in 
common use now. (3) Silicon nitride is not stable enough with respect to hydrolysis in humid atmosphere at high temperature.

In this publication we suggest a method of the solution of these problems by the application of ceramic membranes fixed on the ceramic substrate with holes.

\section{Thin Alumina Films Fabricated by Anodic Oxidation}

Earlier [4], we investigated ceramic MEMS with membranes made of aluminum oxide. These membranes can be fabricated using so-called spark-electrolyte. In this method, aluminum foil with thickness of about 100 microns is put into acidic or basic electrolyte. High pulsing voltage (up to $600 \mathrm{~V}$ ) applied to the aluminum foil leads to local breakdown of the barrier layer. In this spark, aluminum is oxidized at high temperature leading to the formation of poly-crystalline aluminum oxide in $\gamma$-modification. The optimal thickness of the membrane fabricated using spark-electrolyte oxidation of aluminum is of about 20 - 30 microns. In this case, membrane has satisfactory mechanical strength and relatively low heat conductivity in superficial direction assuring good thermal isolation of the heater deposited on top of the membrane. The scheme of this MEMS chip is shown in Fig. 1.

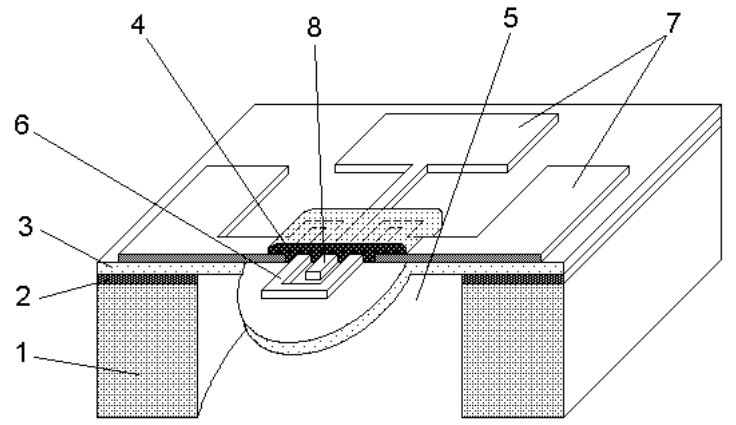

Fig. 1: The scheme of the gas sensor chip made of thin alumina film fabricated by spark-electrolyte oxidation of aluminum. 1 - ceramic substrate; 2 glue; 3 - thin ceramic membrane; 4 - sensing layer; 5 - laser drilled hole; 6 - Pt heater; 7 - contact pads; 8 - measuring electrode for sensing layer.

High porosity of the membrane, in spite of relatively high value of heat transfer coefficient of aluminum oxide $(\sim 25 \mathrm{~W} / \mathrm{m} \cdot \mathrm{K})$ leads to sufficient confinement of the heater temperature. These membranes have sufficient strength for screen printing of the sensing layers on top of the membrane but not sufficient to withstand strong shocks. In addition, the surface is crumbly and rough [4], their roughness exceeds 1 micron, as a result, wire bonding of the sensor chips is difficult, because the tool of ultrasonic wire bonding machine deforms the membrane, and the quality of resulting contact is not perfect.

Another problem cased by roughness of the surface of the membrane is the stability of platinum heater layer deposited on it. To cover all steps on the surface of the membrane, it is necessary to deposit relatively thick (up to 1 micron) layer of platinum. This thick layer, first, increases the cost of the sensor and, second, decreases the reliability of the device because of possible rupture of the platinum line on the steps of alumina film.

To solve this problem, we developed an original method of high-speed galvanostatic anodization of thin aluminum foil in diluted water solution of organic acids. The thickness of foil was in the range between 9 and 14 microns. The optimization of the regimes of oxidation depending on foil size, material and geometry, permitted us to get uniform and high-quality oxidation of whole surface of the foil.

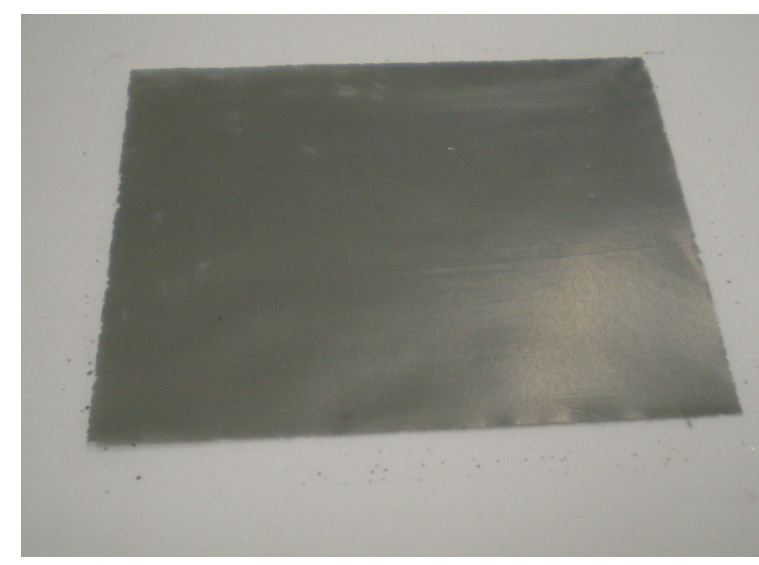

a

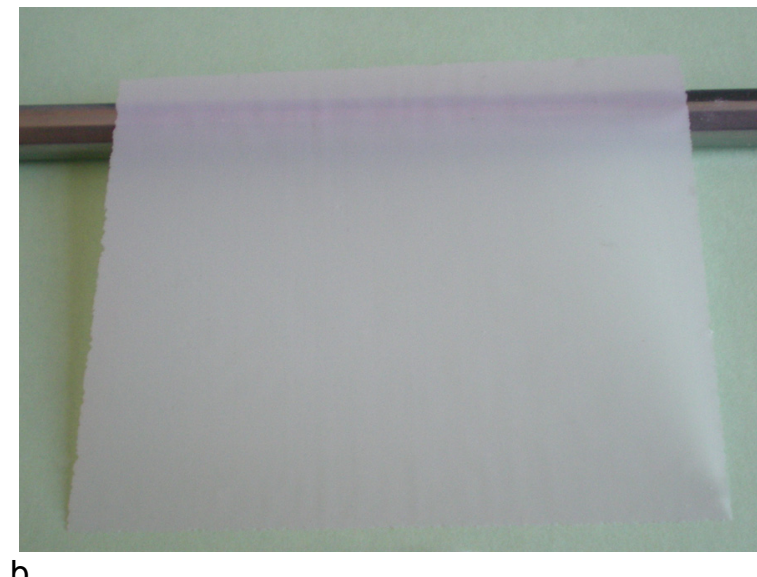

Fig. 2: Thin alumina film madeby anodic oxidation of aluminum foil. The size of the film is of $48 \times 60 \mathrm{~mm}$, their thickness is of 12 microns: (a) the film after oxidation; (b) the film after annealing at $1000^{\circ} \mathrm{C}$ for 15 hours. 
Thermocatalytic and semiconductor gas sensors operate at temperature up to $500^{\circ} \mathrm{C}$. Moreover, the formation of sensing layers of these sensors requires technological annealing of the device at temperature equal to at least $750^{\circ} \mathrm{C}$. Therefore, for proper operation of the sensor, alumina membrane must be annealed before use at high temperature possible.

Photo of these film with thickness of about 12 microns (as prepares and after annealing at $1000^{\circ} \mathrm{C}$ ) are presented in Figs. $2 \mathrm{a}$ and $2 \mathrm{~b}$. The size of the membrane is of $48 \times 60 \mathrm{~mm}$ and equals to the size of typical ceramic substrate used in thick film microelectronics.

The morphology of anodic membranes after annealing at $1150^{\circ} \mathrm{C}$ was studied using scanning electron microscope JEOL JSM 6500. Microphotographs in Fig. 3 show the view of the cross section of the membrane. High uniformity of the surface of the membrane enables further deposition of platinum heater and sensing layer.

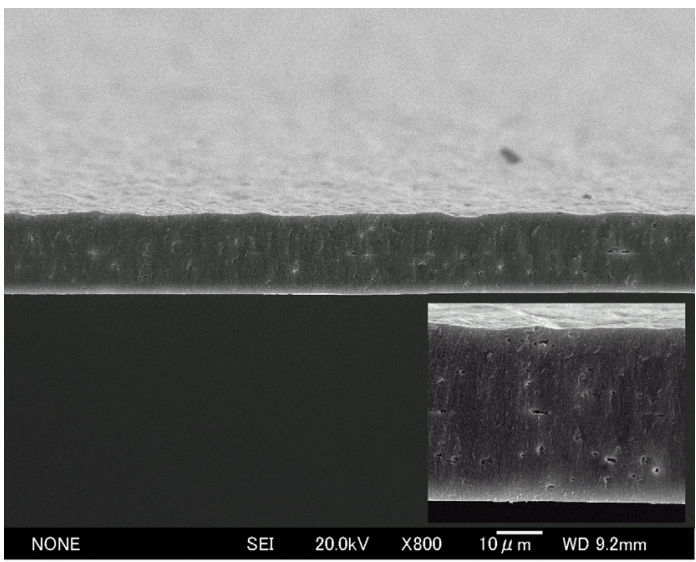

Fig. 3: Cross-section of the 20-micron membrane fabricated by anodic oxidation of aluminum foil. The photo was made after annealing of the membrane at $1150^{\circ} \mathrm{C}$.

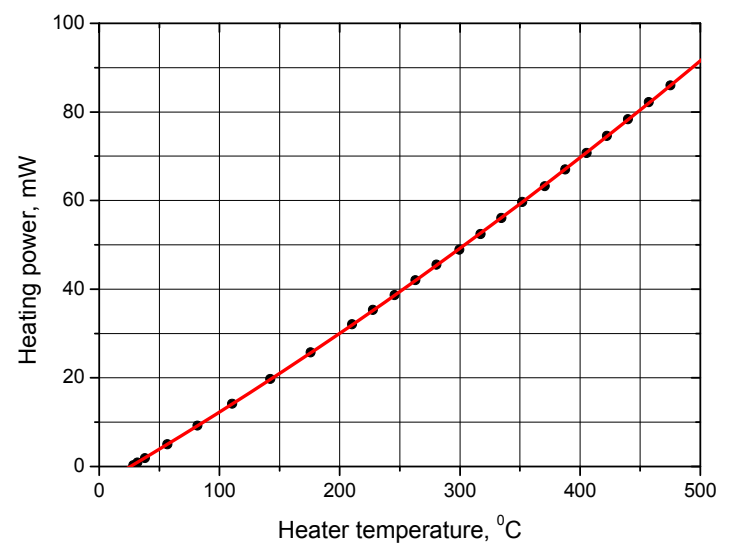

Fig. 4. Heating power of the microhotplate as a function of its temperature. The microhotplate layeout is presented in Fig. 1, the thickness of the alumina film supporting the microhotplate is of about 12 microns.
This alumina film can be glued to the ceramic substrate made of alumina (the scheme of the chip is presented in Fig. 1).

Thermal expansion coefficients of the membrane and the substrate are, evidently, equal to each other. Therefore, thermal treatment of the substrate with the film do not cause cracks in the membrane. Platinum heater is deposited on the membrane by magnetron sputtering. The sensing layer is deposited on the membrane by screen printing; the robustness of the film permits us to do this.

At typical working temperature of $450^{\circ} \mathrm{C}$ necessary for the detection of methane concentration, the microhotplate consumes approximately $80-85 \mathrm{~mW}$ at continuous heating (Fig. 4). For further decrease in power consumption, alumina film can be cut by laser to obtain desirable shape of the microhotplate. Laser cut increases also the stability of the chip at temperature modulation.

The microhotplates based on thin anodic alumina films were also tested for stress stability. For this, a special machine was used, this machine can simulate the shocks with acceleration of about $1000 \mathrm{~m} / \mathrm{s}^{2}$. It was shown that the thick film sensor suspended on platinum wires [5] can withstand $\sim 50$ shocks before the break of wires. The sensor based on alumina membrane withstands more than 10000 shocks before being destroyed.

The microheaters based on anodic alumina films demonstrated perfect stability at pulsing heating. It withstands approximately $6 \cdot 10^{6}$ heating cycles. This number corresponds to approximately 3 years of normal operation with 20-second gas measuring cycle.

\section{MEMS Based on Membranes Made of Yttria Stabilized Zirconia}

We fabricated experimental samples of ceramic MEMS platforms with thin membranes of zirconium dioxide stabilized with yttrium (YSZ). Ceramic film was made by slip casting with consequent annealing under mechanical load. Ceramic slip consists of small particles with size of about $20 \mathrm{~nm}$, therefore satisfactory quality of the film sintering was obtained at relatively low temperature of about $1150^{\circ} \mathrm{C}$ and sintering time of 12 hours. Resulting membrane has thickness of about 10 microns. The top view and the cross section of the membrane are presented in Fig. 5.

After annealing the membrane consists of rounded particles approximately 0.2 micron in diameter, the roughness of the membrane surface does not exceed 0.1 micron. 
YSZ membrane was glued with glass onto the $0.5 \mathrm{~mm}$ thick substrate with $3 \mathrm{~mm}$ holes. The substrate was made of the same material (YSZ) as the membrane. The glass used for gluing the film to the substrate had the same expansion coefficient as the YSZ material.

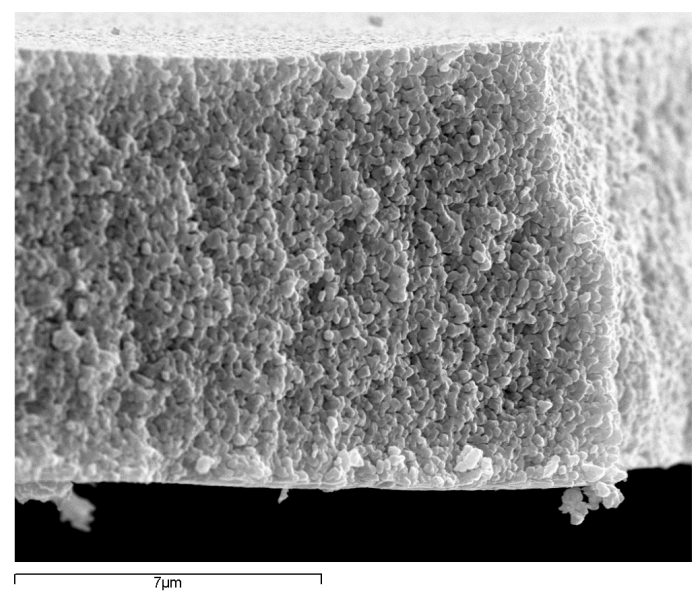

a

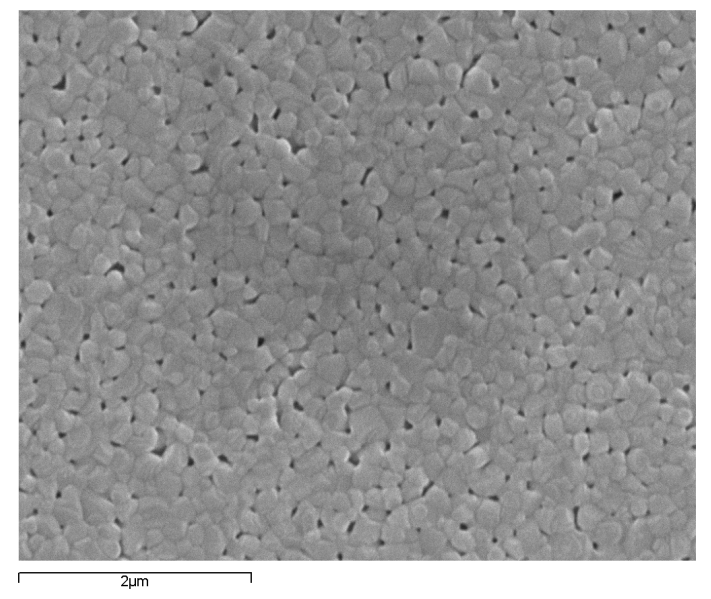

b

Fig. 5: SEM photo of the ceramic membrane made of yttria stabilized zirconia. a. Top view of the membrane; b. cross section (cleaving) of the membrane.

Microheater is fabricated by magnetron sputtering of platinum through shadow mask; the thickness of platinum is of 0.3 micron. After platinum deposition, the substrate with membrane and heaters was annealed at $800^{\circ} \mathrm{C}$ for platinum properties stabilization and cut for the formation of sensor chips with dimension 6 x $6 \mathrm{~mm}$. Layout of the heater is shown in Fig. 1 . The results of the measurement of the I-V characteristics of the microheaters show that the application of YSZ membranes permits us to decrease power consumption of gas sensors down to $75 \mathrm{~mW}$ at continuous heating at $450^{\circ} \mathrm{C}$, and down to approximately $1 \mathrm{~mW}$ and even less at the operation in pulsing heating mode.

\section{Conclusion}

The results described in this paper concerning a comparative study of MEMS platforms of gas sensors containing thin membranes made of annealed anodic alumina and yttria stabilized zirconia (YSZ) show that membranes made of YSZ have much lower heat transfer coefficient in whole temperature range of working temperatures $(25 \mathrm{~W} / \mathrm{m} \cdot \mathrm{K}$ and $2.5 \mathrm{~W} / \mathrm{m} \cdot \mathrm{K}$, respectively). The application of YSZ membranes enables significant decrease in power consumption of the sensor working at $450^{\circ} \mathrm{C}$. This power is equal to about $75 \mathrm{~mW}$ at constant heating of the sensor and to about 1 $\mathrm{mW}$ in average at pulsing heating with duty cycle of 0.01 . Taking into account evident technological and economical advantages of the fabrication of alumina membranes made by anodic oxidation of aluminum foil (technical simplicity, minimal time necessary for membrane fabrication, cheap aluminum foil used in the process, ecological compatibility, etc.) it is necessary to optimize the process of the formation of alumina membrane to minimize their power consumption. Both technologies (anodic alumina and YSZ) can be adapted successfully to mass production of ceramic MEMS platforms of semiconductor and thermocatalytic sensors of combustible gases, gas fire detectors, and electrochemical sensors of oxygen.

\section{Acknowledgements}

The results of this work were obtained partially in the framework of the joint S3 project cofinanced by the program FP-7 of the European Union (grant agreement \# 247768) and Russian ministry of science and education (State contract \# 11.519.11.3006).

\section{References}

[1] A.A.Vasiliev, R.G.Pavelko, et al. $11^{\text {th }}$ International Meeting on Chemical Sensors, Brescia, Italy, July $11-19,2006$, Book of abstracts, p. $127-128$.

[2] http://www.ccmoss.com

[3] http://www.appliedsensor.com

[4] A.A.Vasiliev, R.G.Pavelko, S.Yu.Gogish-Klushin, D.Yu.Kharitonov, O.S.Gogish-Klushina, A.V.Sokolov, A.V.Pisliakov, N.N.Samotaev. Sensors and Actuators B, v. 132 (2008), p. 216223; doi: 0.1016/j.snb.2008.01.043.

[5] A.Vasiliev, R.Pavelko, S.Gogish-Klushin, et al. Sensors based on technology "nano-on-micro" for wireless instruments preventing ecological and industrial catastrophes. In: "Sensors for Environment, health and security", edited by M.I.Baraton, Springer, 2009, p. $205-228$. 